\title{
Cerebral current-source distribution associated with pain improvement by non-invasive painless signaling therapy in patients with failed back surgery syndrome
}

\author{
Chang Han Lee ${ }^{1,3,5}$, Hyeong Seop Kim ${ }^{1}$, Young-Soo Kim ${ }^{2,4,5}$, Seokwon Jung ${ }^{2}$, Chul Ho Yoon ${ }^{1,3,5}$, and \\ Oh-Young Kwon ${ }^{2,4,5}$
}

'Department of Rehabilitation Medicine, Gyeongsang National University Hospital, Jinju, Korea

${ }^{2}$ Department of Neurology, Gyeongsang National University Hospital, Jinju, Korea

${ }^{3}$ Department of Rehabilitation Medicine, Gyeongsang National University College of Medicine, Jinju, Korea

${ }^{4}$ Department of Neurology, Gyeongsang National University College of Medicine, Jinju, Korea

5 Institute of Health Science, Gyeongsang National University College of Medicine, Jinju, Korea

Received March 4, 2021

Revised June 11, 2021

Accepted June 16, 2021

Handling Editor: Won-Hyung Lee

\section{Correspondence}

Oh-Young Kwon

Department of Neurology, Gyeongsang National University Hospital, Gyeongsang National University College of Medicine, 816-15 Jinjudae-ro, Jinju 52727, Korea Tel: +82-55-750-8288

Fax: +82-55-750-1709

E-mail: oykwon@gnu.ac.kr

Chul Ho Yoon

Department of Rehabilitation Medicine, Gyeongsang National University Hospital, Gyeongsang National University College of Medicine, 816-15 Jinjudae-ro, Jinju 52727, Korea

Tel: +82-55-750-8256

Fax: $+82-55-750-8255$

E-mail: yoonch@gnu.ac.kr
Background: Non-invasive painless signaling therapy (NPST) is an electro-cutaneous treatment that converts endogenous pain information into synthetic non-pain information. This study explored whether pain improvement by NPST in failed back surgery syndrome (FBSS) patients is related to cerebral modulation.

Methods: Electroencephalography (EEG) analysis was performed in 11 patients with FBSS. Subjects received daily NPST for 5 days. Before the first treatment, patients completed the Brief Pain Inventory (BPI) and Beck Depression Inventory and underwent baseline EEG. After the final treatment, they responded again to the BPI, reported the percent pain improvement (PPI), and then underwent post-treatment EEG. If the PPI grade was zero, they were assigned to the ineffective group, while all others were assigned to the effective group. We used standardized low-resolution brain electromagnetic tomography (SLORETA) to explore the EEG current-source distribution (CSD) associated with pain improvement by NPST.

Results: The 11 participants had a median age of 67.0 years, and $63.6 \%$ were female. The sLORETA images revealed a beta-2 CSD increment in 12 voxels of the right anterior cingulate gyrus (ACG) and the right medial frontal area. The point of maximal CSD changes was in the right ACG. The alpha band CSD increased in 2 voxels of the left transverse gyrus.

Conclusions: Pain improvement by NPST in FBSS patients was associated with increased cerebral activity, mainly in the right ACG. The change in afferent information induced by NPST seems to be associated with cerebral pain perception.

Key Words: Chronic Pain; Electric Stimulation Therapy; Electroencephalography; Failed Back Surgery Syndrome; Gyrus Cinguli; Information Theory; Neuroimaging; Neuronal Plasticity; Pain Perception. (a) This is an open-access article distributed under the terms of the Creative Commons Attribution Non-Commercial License (http://creativecommons.org/licenses/by-nc/4.0/), which permits unrestricted non-commercial use, distribution, and reproduction in any medium, provided the original work is properly cited.

(C) The Korean Pain Society, 2021
Author contributions: Chang Han Lee: Study conception; Hyeong Seop Kim: Data curation; Young-Soo Kim: Supervision; Seokwon Jung: Investigation; Chul Ho Yoon: Data curation; Oh-Young Kwon: Writing/manuscript preparation. 


\section{INTRODUCTION}

According to one study, the global point prevalence of low back pain was $9.4 \%$ [1]. In adults, the prevalence of chronic back pain is on the rise [2], and as a result the number of surgeries is also increasing [3]. However, surgery may not relieve the pain. Failed back surgery syndrome (FBSS) is one such outcome [4]. For the treatment of FBSS, a type of chronic pain, the first recommendation is conservative management. Surgical consideration is the final stage of the treatment algorithm, but it does not always resolve the pain satisfactorily and involves risks $[5,6]$. Among the modalities of conservative management, drug therapy is the most accessible. However, more than half of patients with chronic pain combine multiple drugs [7]. Such medication polytherapy increases the risk of side effects. Non-invasive painless signaling therapy (NPST) is a treatment modality that provides non-pain information to the cutaneous nerves using patient-specific electrostimulation to control the cerebral perception of pain information. NPST has the advantage of being a minimally invasive non-pharmacological treatment [8-10].

The brain interprets the pain stimuli transmitted from the peripheral nervous system and integrates this interpretation with other stored information. When perceiving pain, the brain employs a process that includes selective attention and intensity coding [11-16]. The pain perceived by the brain may have a different intensity than pain at the peripheral level; pain perception may even occur in the brain in response to non-painful peripheral stimuli [17-20]. The pain matrix is the cerebral network involved in pain perception $[20,21]$. The somatosensory cortex, insula, and anterior cingulate gyrus (ACG) participate in the pain matrix. When the pain matrix is activated, the brain senses and focuses on sensory input from the peripheral system. The brain then integrates the stimuli with the multimodal somatic representation in the cerebral cortex and interprets the integrated information to convert it into a conscious pain perception [20].

Electrical source imaging (ESI) shows the cerebral activity through electroencephalography (EEG) currentsource analysis on magnetic resonance imaging (MRI) matched with a human head model [22-24]. To assess the neurophysiological state and treatment response in cases of chronic pain, the generally accepted test modality has been functional MRI (fMRI). fMRI measures neural brain activity indirectly using the hemodynamic response [2528]. ESI and fMRI complement each other [29]. Although its spatial resolution is inferior to that of fMRI, ESI has the advantage of mapping cerebral neuronal activity directly and at high temporal resolution [22-24,30,31]. Research using ESI to investigate cerebral electrophysiology associ- ated with chronic pain remains rare.

The effects of NPST might be associated with functional modulation of cerebral structures involving pain perception, such as the pain matrix. Our goal was to identify the functional changes in the brain associated with pain improvement by NPST in FBSS patients. For that purpose, using EEG current-source analysis as a frequency domain analysis, we observed the current-source distribution (CSD) difference between FBSS patients with pain improvement by NPST and those with no therapeutic effect.

\section{MATERIALS AND METHODS}

\section{Subject selection}

The Institutional Review Board (IRB) of Gyeongsang National University Hospital approved the present study; the IRB number was 2018-08-016-001. We also registered the study with the World Health Organization International Clinical Trials Registry Platform (WHO ICTRP) through the Clinical Research Information Service, which enables the use of the WHO ICTRP in Korea; the registration number was KCT0003347.

The Department of Rehabilitation Medicine at Gyeongsang University Hospital recruited patients diagnosed with FBSS from November 2018 to June 2019. Patient age was limited to 30-70 years. To avoid difficulties related to cooperation with treatment and evaluation, patients with intellectual disabilities or severe medical, neurological, or psychiatric disorders were excluded. Patients with various conditions that may affect pain sensitivity were also excluded, as were those with a history of ischemic heart disease within 6 months, mesenteric nerve block within 4 weeks, pregnancy or lactation, scars on the back, or chronic use of antiepileptic drugs. Finally, because depressive symptoms can affect pain and brain activity, patients whose degree of depressive symptoms as evaluated by the Beck Depressive Inventory (BDI) was moderate or severe were excluded.

\section{Research process}

After agreeing to participate, enrolled patients visited the hospital every weekday, Monday through Friday, for one week. On Monday, they completed the Brief Pain Inventory (BPI) to determine the baseline pain level and the BDI to assess depressive symptoms. Then, we recorded the patients' first baseline EEGs (time point 1, T1). Next, on the same day, patients received the first session of NPST. They were treated with NPST for 5 days in a row, Monday through Friday. Immediately after the fifth and last thera- 
py session on Friday, they completed the follow-up BPI and reported the percent pain improvement (PPI). After the questionnaires were completed, we recorded the second, post-treatment EEGs (time point 2, T2) for comparison with the baseline T1 EEG.

We then divided the patients into two groups: effective and ineffective groups. The effective group included patients for whom NPST resulted in reduced pain, and the ineffective group included those for whom there was no therapeutic effect. The therapeutic effect was evaluated based on the PPI value reported just after the last (Friday) session of NPST. When evaluating the PPI in terms of grades, a grade of 1 or higher was regarded effective, and a grade of 0 as ineffective.

\section{Questionnaires}

\section{1) BDI}

The BDI is a self-reported inventory and a useful tool for detecting depressive symptoms. It requires that subjects rate their condition during the last week using 21 items describing depressive symptoms. It is a Likert scale-based inventory where each item is given a score from 0 to 3 . The total BDI score combines the 21 items, yielding an overall score of 0-63. Based on the total score, the degrees of depressive symptoms are described as follows: $0-16$, normal; 17-20, mild; 21-23, moderate; and 24-63, severe [32-34].

\section{2) BPI}

The BPI was used to evaluate pain severity [35-37]. It is a Likert scale-based inventory with 11 items that evaluate pain during the last 24 hours. Each of the 11 items is given a score ranging from 0 to 10 . Four items address pain severity, and the other 7 evaluate interference to living functions. In this study, the 4 BPI items addressing pain severity (PSIs-BPI) were used to assess pain severity at two time points: $\mathrm{T} 1$ and $\mathrm{T} 2$. The BPI guides patients to report their pain intensity on a scale from 0 to 10 . A score of 0 indicates "no pain," and a score of 10 means "pain as bad as you can imagine." Total scores on the four-item PSIs-BPI could range from 0-40.

\section{3) PPI}

Patients reported PPI due to NPST immediately after the final treatment session. PPI was categorized into five grades, from grade 0 to grade 4 . The five PPI grades were defined as follows: $0-19 \%$, grade $0 ; 20-39 \%$, grade $1 ; 40$ $59 \%$, grade $2 ; 60-79 \%$, grade 3 ; and $80-100 \%$, grade 4 .

\section{NPST}

The NPST was administered using a Pain Jammer ENS1140 device (ENS System, Bucheon, Korea). The device uses alternating current stimulation, and the maximum current available for treatment was $5.5 \mathrm{~mA}$. The treatment was administered to the area in the low back and buttock region where the patient complained of the most severe pain. The therapist placed four stimulation electrodes along the lines of pain and numbness at the designated treatment location.

After determining the positions of the stimulation electrodes for NPTS, at those spots, we discovered the maximum tolerance intensity of the electric stimulation. Stimulation was started at zero intensity and was then gradually raised until a patient felt a sting or burning sensation. At that point, we added more stimuli with that intensity. If the patient perceived the stimuli as a tolerable tingling sensation by adaptation, we slightly increased the stimulation intensity again. When the patient could not tolerate any more for a certain level of intensity, we slightly reduced the intensity and found the maximum tolerance intensity. NPST was performed using the maximum tolerance intensity, and each treatment session lasted $50 \mathrm{~min}$ utes.

\section{EEG recording}

During EEG recording, patients closed their eyes and remained awake in a calm state. Technicians recorded the EEGs using two digital EEG machines; the recording time was 50 minutes. Two machines were used: a Comet (Grass Telefactor, West Warwick, RI) or a Beehive Horizon (Grass Telefactor). For EEG recording, 25 electrodes were placed on the scalp referencing the International 10-20 system. The positions of the electrodes were as follows: Fp1, F3, C3, P3, O1, Fp2, F4, C4, P4, O2, F7, T7, P7, F8, T8, P8, F9, P9, T9, $\mathrm{F} 10, \mathrm{P} 10, \mathrm{~T} 10, \mathrm{Fz}, \mathrm{Cz}$, and Pz. For the EEG recording, the filter setting was $1-70 \mathrm{~Hz}$ with a notch filter of $60 \mathrm{~Hz}$, and the sampling rate was $400 \mathrm{~Hz}$.

\section{EEG analysis}

We extracted the appropriate EEG segments from the recorded EEGs. Extraction of the EEG segments involved use of the brain electrical source analysis (BESA, v.7.0; MEGIS, Grafelfing, Germany) data review and processing module. The EEG segment duration was 5 seconds, and it was extracted from EEG records with occipital alpha activity and without artifacts. Each patient underwent EEG twice (T1 and T2), and each EEG provided five segments, yielding 10 EEG segments per patient. Among these, 5 were EEG seg- 
ments at $\mathrm{T} 1$, and the other 5 at $\mathrm{T} 2$.

The EEG segments selected through preprocessing were used as data to identify CSD associated with pain improvement by NPST. The current-source analysis proceeded in two steps. The first step involved subtracting the EEG at T1 from the EEG at T2 to obtain the CSD changes induced by NPST in the effective and ineffective groups. The second step entailed subtracting the CSD changes induced by NPST in the ineffective group from those in the effective group to obtain the CSD associated with pain improvement (CSD of effective group [T2 - T1] - CSD of ineffective group [T2 - T1]).

We used standardized low-resolution brain electromagnetic tomography (sLORETA; Key Institute for Brain-Mind Research, Zurich, Switzerland) software to calculate and describe the cerebral CSD. The sLORETA expresses EEG CSD in the brain using a distributed source model. It finds CSD in a three-shell spherical head model registered to the Talairach human brain atlas, converts the CSD found into Montreal Neurological Institute (MNI) coordinates, and represents the transformed coordinates in a digitized MRI from the Brain Imaging Center, MNI. Thus, the sLORETA images obtained through EEG analysis using sLORETA are

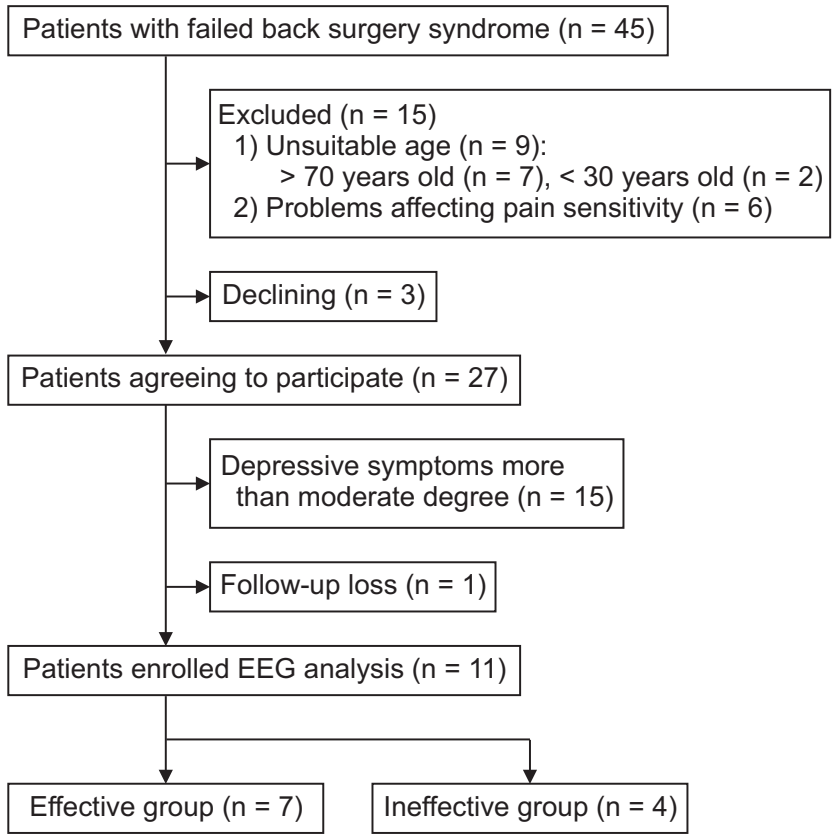

Fig. 1. Flow diagram of patient recruitment. The authors diagnosed 45 patients with failed back surgery syndrome. Fifteen patients of them ousted from the study because of age unsuitability or factors affecting pain sensitivity. Of the remaining 30 patients, 27 patients agreed to participate in the study. The authors also excluded 15 of the 27 patients due to depressive symptoms and lost another patient during followup. Finally, 11 patients were subjects for electroencephalography (EEG) analysis. Seven of them were in the effective group, and the remaining four in the ineffective group. three-dimensional functional images. The images consisted of 6,430 cortical gray voxels, and their spatial resolution was $5 \mathrm{~mm}$ [38].

By performing the current-source analysis, we obtained ESIs in the frequency domain. The seven frequency domains obtained were as follows; $1-3 \mathrm{~Hz}$, delta; 4-7 Hz, theta; 8-12 Hz, alpha; 3-18 Hz, beta-1; $19-21 \mathrm{~Hz}$, beta-2; and $22-30 \mathrm{~Hz}$, beta-3. Statistical significance set at $P<0.05$ (two tailed) expressed the suprathreshold voxels in the sLORETA images.

\section{Statistical analysis for clinical information}

We performed univariate analyses using Mann-Whitney tests for continuous variables and Fisher's exact tests for categorical variables to compare the clinical features between the effective and ineffective groups. R software (version 3.5.3; R Foundation for Statistical Computing, Vienna, Austria) was used for these statistical analyses [39].

\section{RESULTS}

\section{Subject characteristics}

During the study period, 11 of 45 FBSS patients were enrolled in this study (Fig. 1, Table 1). The 11 enrolled participants had a median age of 67.0 years, and 7 (63.6\%) were female. The enrolled FBSS patients had undergone spinal surgery between one and three times. Nine patients had undergone operation twice, and the remaining two patients had undergone surgery three times and once, respectively. Three spinal diseases were the targets of the operations. These include herniated lumbar disc in nine patients, spinal stenosis in seven, and spondylolisthesis in one (Table 1).

Seven of the 11 were classified as belonging to the effective group, and the remaining 4 made up the ineffective group. Table 2 presents a comparison of patients in the effective group with those in the ineffective group. Significant differences were observed between the T1T2 PSIs-BPI scores and in PPI, but no other differences were found (Table 2). The median PPI was $40.0 \%$ and $7.5 \%$ in the effective and ineffective groups, respectively $(P<$ 0.001 ). In terms of the grade of pain improvement, three patients in the effective group had grade 1 improvement, two had grade 2, and the remaining two had grades 3 and 4. All four patients in the ineffective group had grade $0(P$ $=0.030$ ). The median improvement in PSIs-BPI score ( $\mathrm{Tl}-$ T2) was 6.0 points in the effective group and 0.4 points in the ineffective group $(P<0.001)$. 
Table 1. Characteristics of 11 enrolled patients with failed back surgery syndrome treated by noninvasive painless signaling therapy

\begin{tabular}{|c|c|c|c|c|c|c|c|c|c|c|c|}
\hline \multirow{2}{*}{$\begin{array}{l}\text { Recruitment } \\
\text { number }\end{array}$} & \multirow{2}{*}{ Subgroup } & \multirow{2}{*}{ Sex } & \multirow{2}{*}{ Age } & \multicolumn{2}{|c|}{ Spinal surgery } & \multicolumn{3}{|c|}{ Score of PSIs-BPI } & \multicolumn{2}{|c|}{ PPI } & \multirow{2}{*}{ BDI score } \\
\hline & & & & Causes & Number & $\mathrm{T} 1$ & $\mathrm{~T} 2$ & T1-T2 & $\%$ & Grade & \\
\hline 1 & Ineffective & Male & 68 & HLD, SS & 2 & 21 & 21 & 0 & 5 & 0 & 15 \\
\hline 2 & Effective & Male & 65 & SS & 3 & 15 & 10 & 5 & 20 & 1 & 13 \\
\hline 3 & Effective & Male & 59 & HLD & 1 & 14 & 8 & 6 & 40 & 2 & 5 \\
\hline 4 & Effective & Female & 67 & HLD, SS & 2 & 21 & 15 & 6 & 30 & 1 & 11 \\
\hline 5 & Ineffective & Female & 70 & HLD & 2 & 15 & 13 & 2 & 15 & 0 & 13 \\
\hline 6 & Ineffective & Male & 84 & HLD, SS & 2 & 12 & 11 & 1 & 10 & 0 & 8 \\
\hline 7 & Ineffective & Female & 67 & HLD, SS & 2 & 21 & 21 & 0 & 0 & 0 & 16 \\
\hline 8 & Effective & Female & 70 & HLD, SS & 2 & 13 & 9 & 4 & 40 & 2 & 7 \\
\hline 9 & Effective & Female & 67 & SPDL & 2 & 18 & 13 & 5 & 35 & 1 & 15 \\
\hline 10 & Effective & Female & 70 & HLD, SS & 2 & 15 & 8 & 7 & 60 & 3 & 13 \\
\hline 11 & Effective & Female & 55 & HLD & 2 & 22 & 15 & 7 & 80 & 4 & 11 \\
\hline
\end{tabular}

HLD: herniated lumbar disc, SS: spinal stenosis, SPDL: spondylolisthesis, PSIs-BPI: pain severity items of Brief Pain Inventory, PPI: percent of pain improvement, BDI: Beck Depression Inventory, T1: baseline time-point, T2: time-point after the 5th treatment session.

Table 2. Comparisons between the effective group and the ineffective group under noninvasive painless signaling therapy

\begin{tabular}{lccc}
\hline \multicolumn{1}{c}{ Variable } & $\begin{array}{c}\text { Effective group } \\
(\mathrm{n}=7)\end{array}$ & $\begin{array}{c}\text { Ineffective group } \\
(\mathrm{n}=4)\end{array}$ & $P$ value \\
\hline Age (yr) & $67.0(6.5)$ & $69.0(5.8)$ & 0.124 \\
Female & $5(71.4)$ & $2(50.0)$ & 0.477 \\
BDI score & $13.0(4.0)$ & $13.0(3.5)$ & 0.292 \\
Score of PSIs-BPI & & & \\
$\quad$ T1 & $15.0(5.0)$ & $18.0(6.8)$ & 1.000 \\
T2 & $10.0(5.5)$ & $17.0(8.5)$ & 0.105 \\
T1-T2 & $6.0(1.5)$ & $0.5(1.3)$ & 0.008 \\
PPI, \% & $40.0(17.5)$ & $7.5(7.5)$ & 0.008 \\
Grade of PPI & & & 0.027 \\
0 (0-19\%) & $0(0.0)$ & $4(100.0)$ & \\
1 (20-39\%) & $3(42.9)$ & $0(0.0)$ & \\
2 (40-59\%) & $2(28.6)$ & $0(0.0)$ & \\
3 (60-79\%) & $1(14.3)$ & $0(0.0)$ & \\
$4(80-100 \%)$ & $1(14.3)$ & $0(0.0)$ & \\
\hline
\end{tabular}

Values are presented as median (interquartile-range) or number (\%). BDI: Beck Depression Inventory, PSIs-BPI: pain severity items of Brief Pain Inventory, T1: baseline time-point, T2: time-point after the 5th treatment session, PPI: percent of pain improvement.

\section{Changes in current-source density}

In the sLORETA images using a two-tailed test with a significance level of 0.05 , the CSD of the beta- 2 and alpha band significantly increased. The point of maximal CSD change was in the right ACG (Brodmann's area [BA] 32, MNI coordinates: $x=10, y=25, z=25$ ) (Table 3, Fig. 2). No significant CSD changes occurred in the other frequency domains.

The beta-2 CSD increment was observed in 12 suprathreshold voxels of the right hemisphere located in BA 32, the medial part of BA 24, and BA 9 (Table 3, Fig. 2). The three BA locations were the following: BA 32 , the dorso- lateral ACG; BA 24, the ventral ACG; and BA9, the medialand dorsolateral frontal area [40]. Of the 12 suprathreshold voxels, 9 were in the ACG, and 3 in the medial frontal gyrus. The CSD of the alpha band increased in BA 41 in the left hemisphere, with two suprathreshold voxels (Table 3, Fig. 3). BA 41 is a transverse gyrus corresponding to the posterosuperior part of the superior temporal gyrus [40].

\section{DISCUSSION}

This study used the ineffective group as controls. The EEG of each patient was recorded twice, at $\mathrm{T} 1$ and T2. The EEG analysis design for SLORETA image acquisition was the following: CSD of the effective group (T2 - T1) - CSD of the ineffective group (T2 - T1). The CSD difference between the two groups was the primary finding of this study, so the changes in CSD were considered to indicate cerebral activity linked to pain reduction by NPST. As elements of that observation, the CSD of beta-2 and alpha significantly increased in certain cerebral regions. Compared to the alpha band, the CSD change was substantial and broad in the beta- 2 band. The CSD increment locations of the beta2 band were the right ACG gyrus and the right medial frontal area. The CSD increment of the alpha band occurred in the left transverse gyrus. Among the three areas with increased activity, the broadest and most marked increase was in the right ACG. Because the ACG is a component of the pain matrix, the pain matrix could be functionally associated with the therapeutic NPST effect.

The ACG participates in two functional pain perception networks: one of these detects pain information, and the other perceives pain intensity. When these two networks work together as an integrative functional map, people experience subjective pain [11-16]. Because of this pain- 
Table 3. Suprathreshold voxels* for current-source density associated with pain improvement by noninvasive painless signaling therapy

\begin{tabular}{|c|c|c|c|c|c|c|c|c|c|c|c|c|}
\hline \multirow{2}{*}{$\begin{array}{c}\text { Frequency } \\
\text { band }\end{array}$} & \multirow{2}{*}{ Number } & \multicolumn{3}{|c|}{ MNI coordinate } & \multicolumn{3}{|c|}{ Talairach coordinate } & \multirow{2}{*}{$\begin{array}{l}\text { Voxel } \\
\text { value }\end{array}$} & \multirow{2}{*}{$\begin{array}{c}\text { Brodmann } \\
\text { area }\end{array}$} & \multirow{2}{*}{ Side } & \multirow{2}{*}{ Lobe } & \multirow{2}{*}{ Gyrus } \\
\hline & & $x$ & $y$ & z & $x$ & $y$ & z & & & & & \\
\hline \multirow[t]{12}{*}{ Beta-2 } & 1 & 10 & 25 & 25 & 10 & 25 & 22 & 4.28410 & 32 & Right & Limbic lobe & Anterior cingulate \\
\hline & 2 & 10 & 20 & 25 & 10 & 21 & 22 & 4.26373 & 24 & Right & Limbic lobe & Anterior cingulate \\
\hline & 3 & 10 & 20 & 30 & 10 & 21 & 27 & 4.26258 & 24 & Right & Limbic lobe & Anterior cingulate \\
\hline & 4 & 10 & 25 & 30 & 10 & 26 & 26 & 4.26086 & 32 & Right & Limbic lobe & Anterior cingulate \\
\hline & 5 & 15 & 30 & 30 & 15 & 30 & 26 & 4.23470 & 32 & Right & Frontal lobe & Medial frontal \\
\hline & 6 & 15 & 35 & 25 & 15 & 35 & 21 & 4.21994 & 32 & Right & Limbic lobe & Anterior cingulate \\
\hline & 7 & 15 & 35 & 30 & 15 & 35 & 26 & 4.18160 & 9 & Right & Frontal lobe & Medial frontal \\
\hline & 8 & 10 & 30 & 30 & 10 & 30 & 26 & 4.17812 & 32 & Right & Limbic lobe & Anterior cingulate \\
\hline & 9 & 10 & 15 & 30 & 10 & 16 & 27 & 4.12199 & 24 & Right & Limbic lobe & Anterior cingulate \\
\hline & 10 & 10 & 25 & 35 & 10 & 26 & 31 & 4.10583 & 32 & Right & Limbic lobe & Anterior cingulate \\
\hline & 11 & 15 & 35 & 20 & 15 & 35 & 17 & 4.09757 & 32 & Right & Limbic lobe & Anterior cingulate \\
\hline & 12 & 15 & 25 & 35 & 15 & 26 & 31 & 4.08977 & 9 & Right & Frontal lobe & Medial frontal \\
\hline \multirow[t]{2}{*}{ Alpha } & 1 & -40 & -30 & 5 & -40 & -29 & 6 & 4.18309 & 41 & Left & Temporal lobe & Transverse temporal \\
\hline & 2 & -40 & -30 & 10 & -40 & -29 & 11 & 4.12178 & 41 & Left & Temporal lobe & Transverse temporal \\
\hline
\end{tabular}

MNI: Montreal Neurological Institute.

$\star P<0.05(\mathrm{t}=4.083)$, two-tailed.

perceiving network involving the ACG, people sense subjective pain rather than the actual intensity of a noxious stimulus [11].

In the present study, increased activity in the right ACG was associated with pain reduction by NPST. Therefore, it seems that increasing ACG activity may cure chronic pain. It might be possible to correctly code the pain intensity in patients with chronic pain if NPST increases ACG activity. A previous study reported that daily life disruption due to pain declined when the dorsal ACG was stimulated with deep brain stimulation in patients with thalamic pain syndrome [13].

Functional representations associated with pain in the medial frontal cortex are well known. For the representations, pain is localized in the anterior midcingulate cortex, negative emotion in the ventromedial prefrontal cortex, and cognitive control in the dorsal midcingulate cortex. Furthermore, these three representations have a meaningful correlation in the dorsomedial frontal cortex [41]. In the present study, we observed an increase in the beta2 CSD in three suprathreshold voxels of the right medial frontal gyrus. The increase in activity may be significant, considering the functional representations in the medial frontal cortex. Our findings indicate that increased activity was related to pain reduction via NPST.

A pathophysiological alteration in the superior temporal gyrus may occur in patients with postherpetic neuralgia (PHN) [42]. Previous research has revealed that regional homogeneity of the brain increased in PHN patients with effective pain control in some areas, including the right superior temporal gyrus, indicating increased local functional connectivity $[43,44]$. We also observed a CSD change in the superior temporal gyrus, although it was in the opposite hemisphere, and this change was associated with pain reduction in FBSS. Chronic pain may associate with the abnormality of the superior temporal gyrus in both the right and left hemispheres. It seems that pain reduction may result if the areas obtain functional improvement.

The sLORETA model is a distributed ESI model and is free from localization errors compared to the previous version, the LORETA, but it still has spatial resolution limitations [38]. Various factors, such as the number and distribution of electrodes, head models, and EEG-MRI co-registration, affect ESI accuracy $[22,45]$. The localization accuracy of ESI increases as the number of scalp electrodes increases, but accuracy does not continue to improve at more than 89 electrodes. Uniform sampling of the full head surface is required for source localization to work correctly. The accuracy of source localization is comparable between 19 and 46 electrodes when obtaining a LORETA image based on the three-shell spherical head model if the electrodes are distributed across the entire head [22].

The locations of scalp electrodes must be correct to increase the accuracy of source localization in ESI. The individual EEG-MRI co-registration makes it possible to overcome interindividual variation in electrode positions [22]. Selection of head models is another crucial aspect of EEG-MRI co-registration. The spherical model with uniform conductivity is the simplest head model. It is easy to apply and allows rapid calculation, but the human head is not spherical and is not spatially uniform. Realistic head models increase ESI accuracy but take longer to compute $[22,45]$. 


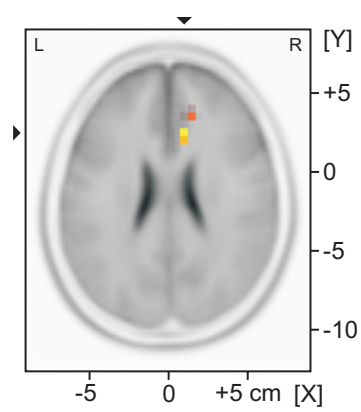

$[X, Y, Z]=[10,25,25][\mathrm{mm}] ;[4.28 \mathrm{E}+0][A-B ; 1,600 \mathrm{~Hz}]$

SLORETA
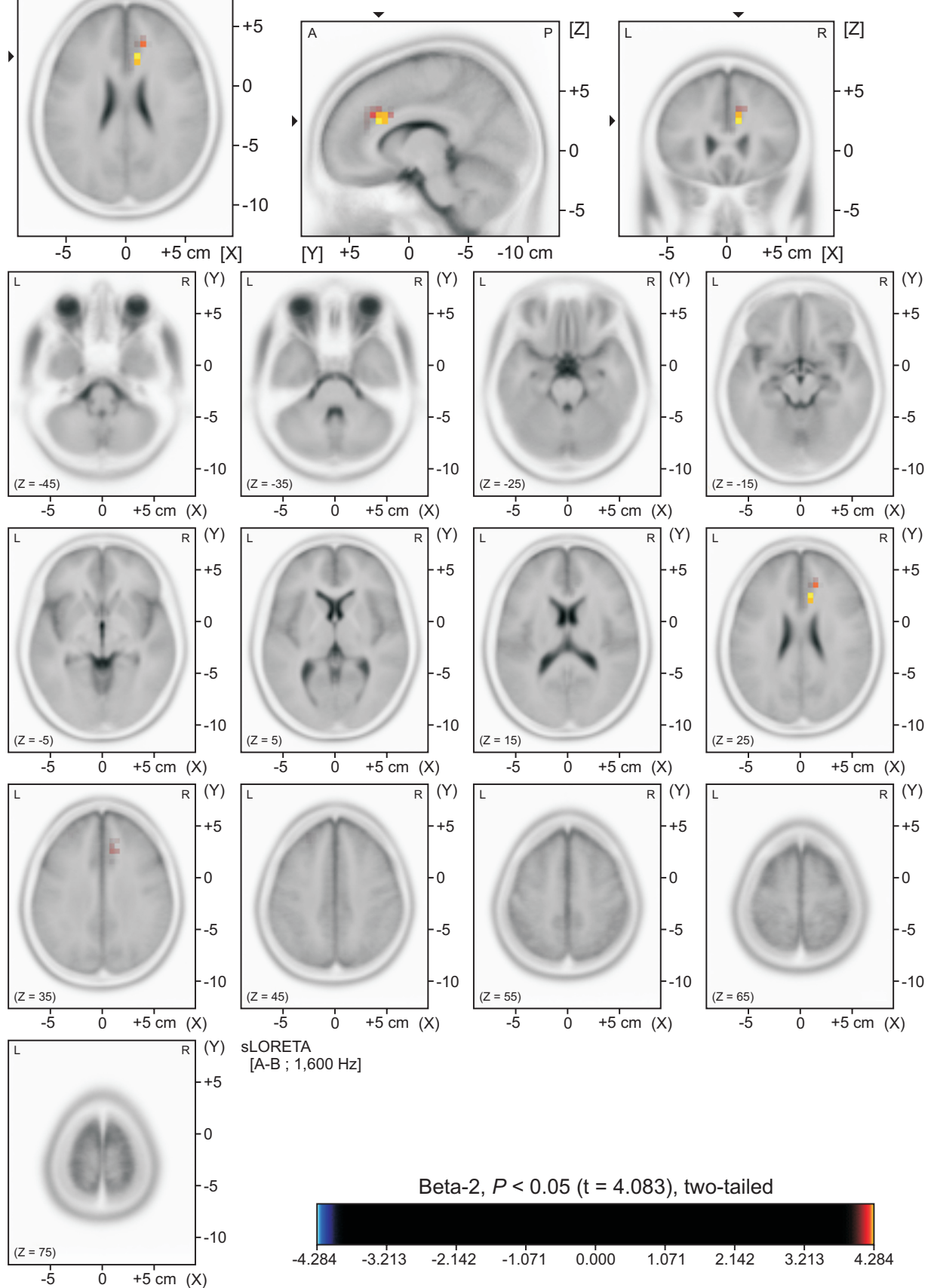
sLORETA
[A-B ; 1,600 Hz]

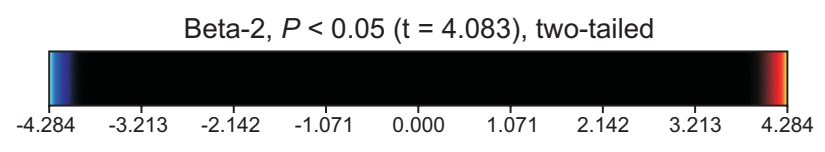

Fig. 2. Standardized low-resolution brain electromagnetic tomography (sLORETA) images showing beta-2 current-source distribution (CSD) associated with pain improvement by non-invasive painless signaling therapy. The beta-2 CSD increment was in the right anterior cingulate gyrus and the right medial frontal area. The number of suprathreshold voxels was 12 $(P<0.05$, two-tailed). The location of the maximal point of CSD changes was the right anterior cingulate gyrus (Brodmann's area 32, Montreal Neurological Institute coordinates: $x=10, y=25, z=25$ ).

ESI serves as an adjunctive modality that finds the epileptogenic zone [46]. Precise localization is required when performing ESI for presurgical assessment, and the ESI used in the present study ( 25 electrodes and based on a 3 -shell spherical head model) would be insufficient for this task. However, our goal was to investigate cerebral structures associated with NPST-induced pain relief in FBSS patients. The resolution of the sLORETA imaging obtained in this study may be sufficient to discriminate among the brain structures. In any case, the findings of this study help clarify the neurophysiological mechanisms of FBSS pain.

This study involved some limitations. The first weakness was the small number of enrolled patients. However, the findings are of value because we observed direct neural activity using ESI. Few researchers have observed ESI associated with the therapeutic effect of NPST in FBSS. The second limitation was the short-term follow-up, which made it difficult to know how long the observed CSD changes might persist. More research is needed with larger populations of patients with FBSS to explore whether our results can be reproduced, and long-term follow-up re- 


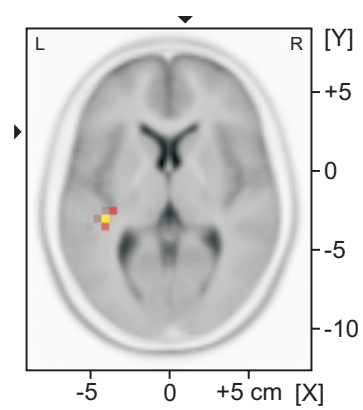

$[X, Y, Z]=[-40,-30,5][\mathrm{mm}] ;[4.18 \mathrm{E}+0] \quad[A-B ; 800 \mathrm{~Hz}]$

SLORETA
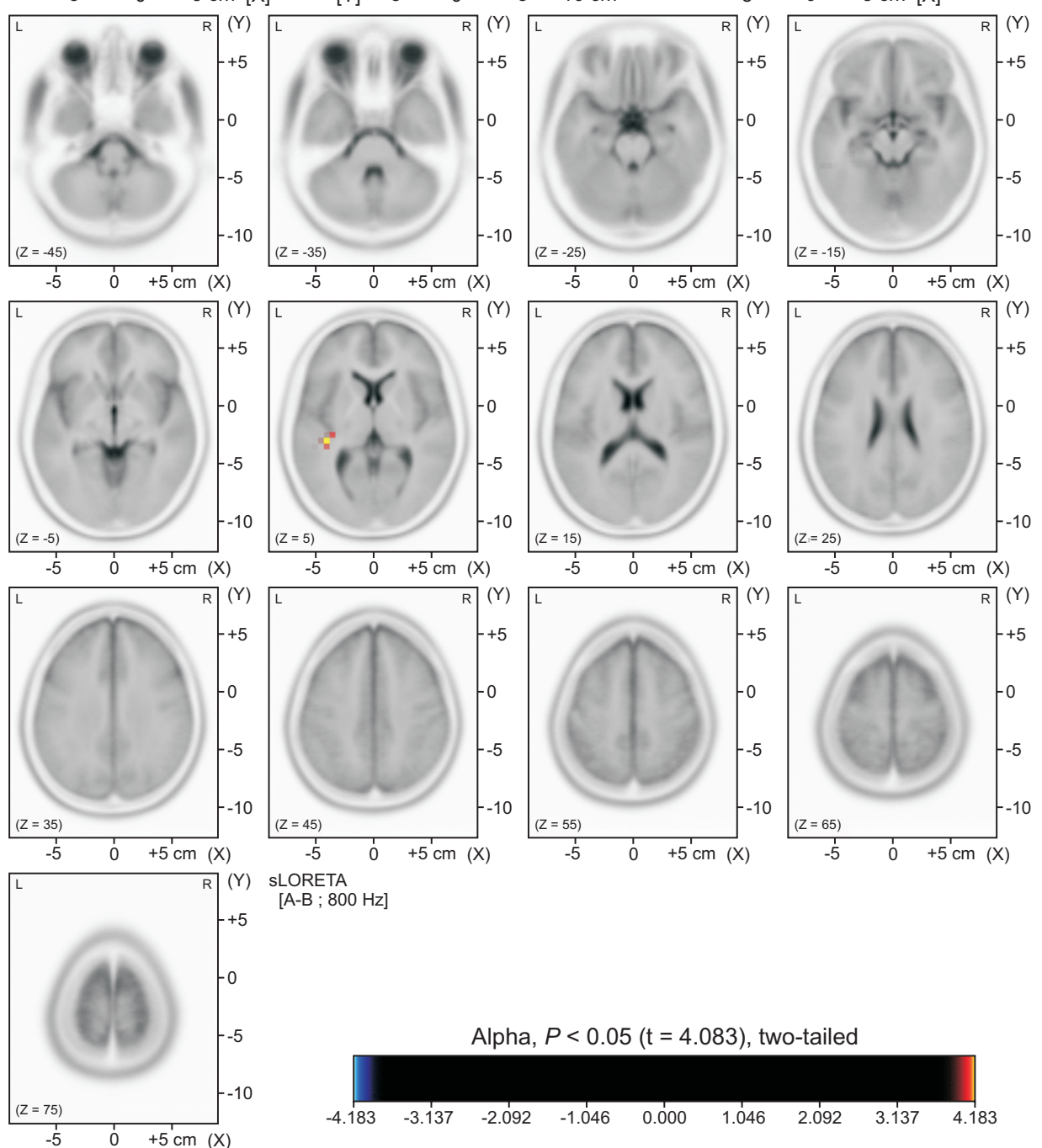
sLORETA
[A-B ; $800 \mathrm{~Hz}$

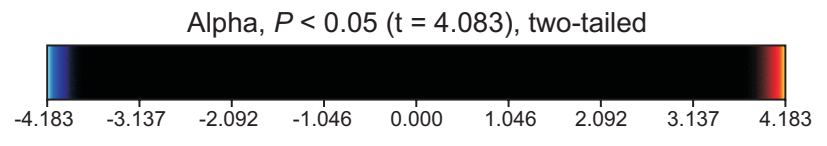

Fig. 3. Standardized low-resolution brain electromagnetic tomography (sLORETA) images showing alpha current-source distribution (CSD) associated with pain improvement by non-invasive painless signaling therapy. The alpha CSD increment was in the left transverse gyrus of the temporal lobe. The number of suprathreshold voxels was two $(P<0.05$, twotailed).

search is needed to further evaluate the long-term effects of NPST. Another drawback is that this is an observational study rather than an experimental one, so that it is unable to examine the causal relationship between pain reduction and CSD changes. The interpretation that there is an association between them, on the other hand, maybe appropriate. The present authors excluded patients taking antiepileptic drugs chronically from this study. This exclusion could be a drawback if this study aimed to observe the effectiveness of NPST in FBSS patients. Rather than that, the purpose of this study was to research cerebral functional changes associated with NPST-induced pain improvement in FBSS patients.

Certain brain structures, such as the pain matrix, serve to detect and perceive pain. The brain also has the plasticity to adapt to alterations of afferent peripheral stimulus information. NPST modifies such afferent information. In this study, the pain reduction induced by NPST in patients with FBSS was associated with increased cerebral activity, mainly in the right ACG. The ACG is a cerebral area that is included in the pain matrix. Thus, NPST may generate therapeutic effects by affecting the pain matrix. 


\section{CONFLICT OF INTEREST}

No potential conflict of interest relevant to this article was reported.

\section{FUNDING}

No funding to declare.

\section{ORCID}

Chang Han Lee, https://orcid.org/0000-0001-8351-5226 Hyeong Seop Kim, https://orcid.org/0000-0001-5022-6030 Young-Soo Kim, https://orcid.org/0000-0003-1040-372X Seokwon Jung, https://orcid.org/0000-0001-6134-1173 Chul Ho Yoon, https://orcid.org/0000-0003-4916-4180 Oh-Young Kwon, https://orcid.org/0000-0001-9576-1926

\section{REFERENCES}

1. Hoy D, March L, Brooks P, Blyth F, Woolf A, Bain C, et al. The global burden of low back pain: estimates from the Global Burden of Disease 2010 study. Ann Rheum Dis 2014; 73: 96874.

2. Smith M, Davis MA, Stano M, Whedon JM. Aging baby boomers and the rising cost of chronic back pain: secular trend analysis of longitudinal Medical Expenditures Panel Survey data for years 2000 to 2007. J Manipulative Physiol Ther 2013; 36: 2-11.

3. Rajaee SS, Bae HW, Kanim LE, Delamarter RB. Spinal fusion in the United States: analysis of trends from 1998 to 2008. Spine (Phila Pa 1976) 2012; 37: 67-76.

4. Harvey AM. Classification of chronic pain-descriptions of chronic pain syndromes and definitions of pain terms. Clin J Pain 1995; 11: 163.

5. Diebo BG, Passias PG, Marascalchi BJ, Jalai CM, Worley NJ, Errico TJ, et al. Primary versus revision surgery in the setting of adult spinal deformity: a nationwide study on 10,912 patients. Spine (Phila Pa 1976) 2015; 40: 1674-80.

6. Baber Z, Erdek MA. Failed back surgery syndrome: current perspectives. J Pain Res 2016; 9: 979-87.

7. Freynhagen R, Bennett MI. Diagnosis and management of neuropathic pain. BMJ 2009; 339: b3002.

8. Ricci M, Pirotti S, Scarpi E, Burgio M, Maltoni M, Sansoni E, et al. Managing chronic pain: results from an open-label study using MC5-A Calmare ${ }^{\circledR}$ device. Support Care Cancer 2012; 20: 405-12.

9. Smith TJ, Coyne PJ, Parker GL, Dodson P, Ramakrishnan V. Pilot trial of a patient-specific cutaneous electrostimulation device (MC5-A Calmare ${ }^{\odot}$ ) for chemotherapy-induced peripheral neuropathy. J Pain Symptom Manage 2010; 40: 883-91.

10. Shannon CE. A mathematical theory of communication. Bell Syst Tech J 1948; 27: 379-423.

11. Craig AD, Reiman EM, Evans A, Bushnell MC. Functional imaging of an illusion of pain. Nature 1996; 384: 258-60.

12. Foltz EL, White LE Jr. Pain "relief" by frontal cingulumotomy. J Neurosurg 1962; 19: 89-100.

13. Levi V, Cordella R, D’Ammando A, Tringali G, Dones I, Messina $\mathrm{G}$, et al. Dorsal anterior cingulate cortex (ACC) deep brain stimulation (DBS): a promising surgical option for the treatment of refractory thalamic pain syndrome (TPS). Acta Neurochir (Wien) 2019; 161: 1579-88.

14. Peyron R, García-Larrea L, Grégoire MC, Costes N, Convers $\mathrm{P}$, Lavenne F, et al. Haemodynamic brain responses to acute pain in humans: sensory and attentional networks. Brain 1999; 122(Pt 9): 1765-80.

15. Sikes RW, Vogt BA. Nociceptive neurons in area 24 of rabbit cingulate cortex. J Neurophysiol 1992; 68: 1720-32.

16. Vaccarino AL, Melzack R. Analgesia produced by injection of lidocaine into the anterior cingulum bundle of the rat. Pain 1989; 39: 213-9.

17. Baumgärtner U, Tiede W, Treede RD, Craig AD. Laser-evoked potentials are graded and somatotopically organized anteroposteriorly in the operculoinsular cortex of anesthetized monkeys. J Neurophysiol 2006; 96: 2802-8.

18. Hofbauer RK, Fiset P, Plourde G, Backman SB, Bushnell MC. Dose-dependent effects of propofol on the central processing of thermal pain. Anesthesiology 2004; 100: 386-94.

19. Lee MC, Mouraux A, Iannetti GD. Characterizing the cortical activity through which pain emerges from nociception. J Neurosci 2009; 29: 7909-16.

20. Legrain V, Iannetti GD, Plaghki L, Mouraux A. The pain matrix reloaded: a salience detection system for the body. Prog Neurobiol 2011; 93: 111-24.

21. Peyron R, Laurent B, García-Larrea L. Functional imaging of brain responses to pain. A review and meta-analysis (2000). Neurophysiol Clin 2000; 30: 263-88.

22. Michel CM, Murray MM, Lantz G, Gonzalez S, Spinelli L, Grave de Peralta R. EEG source imaging. Clin Neurophysiol 2004; 115: 2195-222.

23. He B, Sohrabpour A, Brown E, Liu Z. Electrophysiological source imaging: a noninvasive window to brain dynamics. Annu Rev Biomed Eng 2018; 20: 171-96.

24. Gross J, Kujala J, Hämäläinen M, Timmermann L, Schnitzler A, Salmelin R. Dynamic imaging of coherent sources: studying neural interactions in the human brain. Proc Natl Acad Sci U S A 2001; 98: 694-9.

25. Davis KD, Flor H, Greely HT, Iannetti GD, Mackey S, Ploner M, et al. Brain imaging tests for chronic pain: medical, legal and ethical issues and recommendations. Nat Rev Neurol 2017; 13: 624-38. 
26. Tracey I, Johns E. The pain matrix: reloaded or reborn as we image tonic pain using arterial spin labelling. Pain 2010; 148: 359-60.

27. Bélanger M, Allaman I, Magistretti PJ. Brain energy metabolism: focus on astrocyte-neuron metabolic cooperation. Cell Metab 2011; 14: 724-38.

28. Logothetis NK. The underpinnings of the BOLD functional magnetic resonance imaging signal. J Neurosci 2003; 23: 3963-71.

29. Nguyen T, Potter T, Karmonik C, Grossman R, Zhang Y. Concurrent EEG and functional MRI recording and integration analysis for dynamic cortical activity imaging. J Vis Exp 2018; 136: 56417.

30. Seeber M, Cantonas LM, Hoevels M, Sesia T, Visser-Vandewalle V, Michel CM. Subcortical electrophysiological activity is detectable with high-density EEG source imaging. Nat Commun 2019; 10: 753.

31. Ramon C, Schimpf PH, Haueisen J. Influence of head models on EEG simulations and inverse source localizations. Biomed Eng Online 2006; 5: 10.

32. Beck AT, Ward CH, Mendelson M, Mock J, Erbaugh J. An inventory for measuring depression. Arch Gen Psychiatry 1961; 4: 561-71.

33. Kwon OY, Park SP. Frequency of affective symptoms and their psychosocial impact in Korean people with epilepsy: a survey at two tertiary care hospitals. Epilepsy Behav 2013; 26 : 51-6.

34. Rhee MK, Lee YH, Park SH, Sohn CH, Chung YC, Hong SK, et al. A standardization study of Beck Depression Inventory 1 - Korean version (K-BDI): reliability and factor analysis. Korean J Psychopathol 1995; 4: 77-95.

35. Cleeland CS, Ladinsky JL, Serlin RC, Nugyen CT. Multidimensional measurement of cancer pain: comparisons of US and Vietnamese patients. J Pain Symptom Manage 1988; 3: 23-7.
36. Cleeland C. Research in cancer pain. What we know and what we need to know. Cancer 1991; 67(3 Suppl): 823-7.

37. Yun YH, Mendoza TR, Heo DS, Yoo T, Heo BY, Park HA, et al. Development of a cancer pain assessment tool in Korea: a validation study of a Korean version of the brief pain inventory. Oncology 2004; 66: 439-44.

38. Pascual-Marqui RD. Standardized low-resolution brain electromagnetic tomography (sLORETA): technical details. Methods Find Exp Clin Pharmacol 2002; 24 Suppl D: 5-12.

39. R Core Team. R: a language and environment for statistical computing [Internet]. Vienna: R Foundation for Statistical Computing; 2020. Available at: https://www.R-project.org/.

40. SimićG, Hof PR. In search of the definitive Brodmann's map of cortical areas in human. J Comp Neurol 2015; 523: 5-14.

41. Kragel PA, Kano M, Van Oudenhove L, Ly HG, Dupont P, Rubio A, et al. Generalizable representations of pain, cognitive control, and negative emotion in medial frontal cortex. Nat Neurosci 2018; 21: 283-9.

42. Zhang Y, Yu T, Qin B, Li Y, Song G, Yu B. Microstructural abnormalities in gray matter of patients with postherpetic neuralgia: a diffusional kurtosis imaging study. Pain Physician 2016; 19: E601-11.

43. Jiang L, Zuo XN. Regional homogeneity: a multimodal, multiscale neuroimaging marker of the human connectome. Neuroscientist 2016; 22: 486-505.

44. Zhang Y, Cao S, Yuan J, Song G, Yu T, Liang X. Functional and structural changes in postherpetic neuralgia brain before and six months after pain relieving. J Pain Res 2020; 13: 90918.

45. Koles ZJ. Trends in EEG source localization. Electroencephalogr Clin Neurophysiol 1998; 106: 127-37.

46. Sharma P, Seeck M, Beniczky S. Accuracy of interictal and ictal electric and magnetic source imaging: a systematic review and meta-analysis. Front Neurol 2019; 10: 1250. 